\title{
Correlação entre exposição ao óxido de etileno e câncer de próstata: doença ocupacional?
}

\section{Correlation between prostatic cancer and ethylene oxide exposure: occupational disease?}

\author{
Ricardo Ribeiro Peixinhoㅜ ${ }^{\text {; Carmen Silvia M.G. Miziara }}{ }^{2}$
}

Peixinho RR; Miziara CSMG. Correlação entre exposição ao óxido de etileno e câncer de próstata: doença ocupacional? Saúde, Ética \& Justiça. 2013;18(2):167-72.

\begin{abstract}
RESUMO: Estudos publicados na literatura médica tentam demonstrar a relação entre a exposição ocupacional a agentes químicos e o aumento de incidência de câncer de próstata. A doença é de etiologia multifatorial, envolvendo fatores genéticos, ambientais, a idade, a etnia, a história familiar e a dieta. O objetivo desse estudo foi verificar a possibilidade da associação entre a exposição laboral ao óxido de etileno e o desenvolvimento de câncer de próstata para o estabelecimento do nexo causal ou da concausa por meio de revisão de literatura nas principais bases de dados disponíveis dos últimos 20 anos. A conclusão desse estudo foi que ainda não existem evidências consistentes de que qualquer agente químico tenha influência no aparecimento do câncer de próstata. Consequentemente, até a presente data, não há como se atribuir ao óxido de etileno nexo de causalidade ou de concausalidade para o desenvolvimento dessa doença.
\end{abstract}

PALAVRAS-CHAVE: Neoplasias da próstata/epidemiologia; Fatores de risco; Óxido de etileno; Exposição ocupacional; Câncer ocupacional; Riscos ocupacionais.

1. Aluno da pós-graduação do curso de Perícias Médicas e Medicina Legal da Faculdade de Ciências Médicas da Santa Casa de São Paulo

2. Departamento de Medicina Legal, Ética Médica, Medicina Social e do Trabalho da Faculdade de Medicina da Universidade de São Paulo.

Endereço para correspondência: Rua Herculano do Livramento 120 ap. 602. Monte Alto, SP - CEP: 15910-000 e-mail: ribeiropeixinho@uol.com.br; csmgm@uol.com.br 


\section{INTRODUÇ̃̃̃O}

$\mathbf{O}$ câncer de próstata $(\mathrm{CP})$ é a segunda maior causa de óbito em homens adultos dentre as doenças neoplásicas malignas no Brasil, sendo superado apenas pelo câncer de pulmão. ${ }^{1}$. O aumento das taxas de incidencia do $\mathrm{CP}$ pode ser parcialmente justificado pela evolução dos métodos diagnósticos, pela melhoria da qualidade dos sistemas de informação no país ou pelo aumento de expectativa de vida pelo brasileiro ${ }^{2}$. Apesar de ser doença de etiologia multifatorial, alguns fatores de risco são bem definidos para o desenvolvimento de $\mathrm{CP}$, entre eles, temos: idade avançada, etnia, predisposição familiar e dieta ${ }^{3,4}$.

$\mathrm{O}$ envelhecimento é considerado o fator de risco mais significante. A incidência do $\mathrm{CP}$ em homens com idade superior a 50 anos é $30 \%$ maior que nos indivíduos em idade mais jovem, e vai aumentando progressivamente até alcançar a proporção de $50 \%$ aos 80 anos $^{5}$. Em relação à etnia, os afroamericanos têm maior risco de desenvolver a doença, entre dez a quarenta vezes mais que os asiáticos ${ }^{6,7}$. Parentes de primeiro grau de sujeitos com $\mathrm{CP}$ apresentam de duas a três vezes mais chance de ter a doença, quando comparados a homens na população geral ${ }^{8,9}$. A dieta altamente calórica e os hormônios masculinos também são fatores de risco ${ }^{10,11}$. Dado o exposto, estima-se que um em cada dez homens, durante a vida, desenvolverá carcinoma de próstata clinicamente evidente ${ }^{12}$. A incidência é subestimada, pois muitos tumores permanecem assintomáticos, sendo diagnosticados apenas em procedimentos de necropsia ${ }^{13}$. Estudos necroscópicos revelaram que $30 \%$ dos homens acima de 50 anos, os quais morreram por outras causas, apresentavam focos de adenocarcinoma na próstata ${ }^{14}$. Existem na literatura médica vários trabalhos que buscam a correlação entre o $\mathrm{CP}$ e fatores de risco ocupacionais relacionados a agentes químicos como os pesticidas, o álcool, o cromo, a fabricação de pneus e a borracha ${ }^{15-19}$ e estudos que buscam agentes que poderiam reduzir o desenvolvimento desse câncer ${ }^{20,21}$. Porém, até a presente data, não se conseguiu confirmação de nenhum desses fatores ou agentes em trabalhos randomizados.

$\mathrm{O}$ presente relato de caso foi realizado a partir de um processo trabalhista no qual o trabalhador de empresa de produtos químicos, diagnosticado com $\mathrm{CP}$, alegou que a sua doença tinha nexo ocupacional por exposição ao óxido de etileno $(\mathrm{OEt})$. A discussão do laudo foi embasada em dados na literatura médica obtida nas principais bases de dados de acesso livre. Dentre essas bases foram selecionadas: PubMed, PMC, Scielo, Capes periódicos e Biblioteca USP Sibinet. Os descritores atilizados foram: prostatic cancer; epidemiology, risk factor, ethylene oxide; occupational exposure; occupational cancer; occupational risks.

\section{Relato de caso médico-pericial}

O autor, nascido em agosto de 1953, raça branca, engenheiro, moveu ação trabalhista contra a empresa em que trabalhou durante 30 anos (de $1^{\circ}$ de junho de 1978 até 14 de fevereiro de 2008). Alegou ter adquirido CP em decorrência de exposição a agentes químicos, particularmente ao OEt. Referiu o autor que desde sua admissão até o ano de 1995 trabalhou exposto ao OE em razão de atividades de descarga e estocagem da substância a granel e da localização de seu posto de trabalho. Em 2007, foi detectado nível elevado de PSA (prostate-specific-antigen) por exame laboratorial utilizado para rastreamento de CP. Relatou que a biopsia confirmou a neoplasia maligna. Acrescentou, ainda, que seu médico assistente indicou tratamento cirúrgico e prescreveu Goserelinacetate (Zoladex ${ }^{\circledR}$ ) que é uma substância análoga ao LH-RH (luteinising hormone-releasing hormone) mensalmente. Em uso dessa medicação, consultou outros profissionais das especialidades de oncologia, urologia e radioterapia, e optou por se submeter a tratamento radioterápico apenas. Como evento adverso ao tratamento, apresentou incontinência urinária transitória, que foi superada com fisioterapia. Não fez qualquer outro tipo de tratamento medicamentoso complementar e seus exames de controle (dosagens do PSA) têm-se mostrado dentro dos padrões de referência (normais). Negou tabagismo em qualquer tempo. Faz acompanhamento dietético com nutricionista e pratica esporte (natação e corrida). Referiu câncer ósseo acometendo genitora e câncer cerebral em tia materna. Negou antecedente familiar de CP. Submeteu-se anualmente a exames preventivos com objetivo de preservar a saúde (check-up) utilizando o plano de saúde da empresa onde laborou, entre os quais dosagem bioquímica de fosfatase ácida como triagem de comprometimento tumoral da próstata. Em 12/12/2006, os resultados dos exames de fosfatase ácida total e fosfatase ácida fração prostática estavam dentro dos limites normais. Na avaliação pericial não foram observadas anormalidades ao exame físico.

Os exames complementares apresentados no ato pericial foram: US Próstata (04/05/2007): imagem nodular sólida hipoecogênica na zona periférica da região média esquerda de $1,2 \mathrm{~cm}$.; anátomo patológico da biópsia prostática $(08 / 05 / 2007)$ : adenocarcinoma grau 7 (4+3) de Gleason.; cintilografia óssea (24/05/2007): hipercaptação na parte inferior do íleo, acetábulo, púbis e ísquio à esquerda; não se pode excluir a presença de metástases; Cintilografia óssea (18/05/2009): a alteração na hemibacia esquerda caracterizada por aumento de intensidade de captação do rádio fármaco e da extensão e área focal hipercaptante na porção póstero-inferior do ísqueo direito. Essas alterações podem representar remodelação traumática, mas não é possível excluir 
metátase óssea; Cintilografia óssea (12/06/2010): a área focal hipercaptante na porção póstero-inferior do ísquio direito apresentou discreta diminuição da captação.

\section{DISCUSSÃO}

O Óxido de etileno (OEt) $\left(\mathrm{C}_{2} \mathrm{H}_{4} \mathrm{O}\right)$ é um gás inflamável e incolor em temperaturas acima de $51.3^{\circ} \mathrm{F}$ $\left(10.7{ }^{\circ} \mathrm{C}\right)$, pertencente ao grupo epóxido, que pode ser encontrado sob as diferentes denominações, como as descritas na chemical abstracts service (CAS 75-218). Possui valor limite de tolerância determinado pela ACGIH (American Conference of Industrial Hygienists) de $1 \mathrm{ppm}$, sendo esse o nível de OEt a que o trabalhador pode permanecer exposto durante oito horas diárias e 40 horas semanais. É um produto utilizado principalmente em indústrias químicas como intermediário na produção de etilenoglicol e como agente esterilizante de equipamentos médicos. De acordo com a Occupational Safety and Health Administration (OSHA), o OEt é uma sustância considerada com potencial carcinogênico, sobretudo em relação ao sistema hematopoiético ${ }^{22}$, mas nenhum estudo até a presente data foi conclusivo ${ }^{8-10,22-}$ ${ }^{24}$. O risco de desenvolvimento de doença neoplásica maligna associada à exposição ao OEt não pode, até a presente data, ser confirmado. Um estudo de coorte analisou 29.800 trabalhadores expostos ao OEt, e 2.540 óbitos e concluiu que não foi possível confirmar a existência de maior incidência de leucemia, linfoma não Hodgkin, câncer de estômago, câncer de pâncreas ou de cérebro nesse grupo de pessoas ${ }^{25,26}$.

Em 2011, Mikoczy et al. analisaram a incidência de câncer entre trabalhadores expostos ao OEt e concluíram que a incidência de câncer ocupacional é baixa, mas não concluíram sobre a possibilidade de aumento de risco de câncer de mama ${ }^{26}$.

A abordagem da associação de CP à exposição ocupacional a agentes químicos é muito presente na literatura médica, porém nenhum estudo foi conclusivo ${ }^{5,6,14}$. Existem vários estudos que tentam comprovar a ingestão de quantidades aumentadas de Cádmio (Cd) como fator de risco para o surgimento da neoplasia maligna de próstata ${ }^{27,28}$. Todavia, um trabalho que incluiu os artigos publicados no MEDLINE de 1966 a 2002, buscando essa associação concluiu que os estudos epidemiológicos não confirmaram que o cadmio seja uma causa de CP, sugerindo novos estudos para a discrepância entre achados laboratoriais e epidemiológicos ${ }^{29}$.

Estudos americanos e europeus demonstraram a presença de maior risco de $\mathrm{CP}$ em trabalhadores na agricultura em relação à população geral. Entretanto, não foi possível demonstrar seguramente a associação entre pesticidas ou agentes químicos de grupos similares e $\mathrm{CP}^{30,31}$.
O CP é uma das neoplasias malignas mais frequentes na população masculina e apresenta caráter multifatorial. Nos Estados Unidos (EUA) representa $25 \%$ de todos os tumores malignos diagnosticados no sexo masculino, sendo estimado que mais de 32.000 indivíduos morrerão em decorrência da doença ${ }^{32}$. No Brasil, segundo o INCA, espera-se que mais de 60.000 novos casos sejam diagnosticados no ano de 2012, portanto, semelhante ao que se observa nos EUA, é a neoplasia mais frequente em homens brasileiros, sendo semente superada pelo câncer de pele não melanoma ${ }^{33}$.

$O$ fator racial exerce importante influência, pois indivíduos de raça negra apresentam maior risco de desenvolverem CP, tumores mais agressivos e com início mais precoce, quando comparados com a população geral ${ }^{34}$. Os fatores determinantes do risco de desenvolvimento de CP clínico ainda permanecem desconhecidos, embora existam três fatores de risco bem definidos: idade avançada, etnia e fatores hereditários ${ }^{35}$. A presença de um familiar de primeiro grau com história de doença (CP) determina a duplicação de risco; quando são dois ou mais familiares afetados, o risco aumenta entre cinco e 11 vezes $^{36}$. Aproximadamente, 9\% dos indivíduos com $\mathrm{CP}$ possui $\mathrm{CP}$ hereditário genuíno, definido como três ou mais familiares afetados, ou como mínimo de dois familiares afetados com idade inferior a 55 anos. A carga hereditária é imutável e não há como evitar o desenvolvimento desse tumor em indivíduo geneticamente predisposto ${ }^{3,4}$.

O Ministério da Saúde e Consumo-Espanha, 2003, considerou a existência de evidência suficiente para classificar o OEt como cancerígeno em humanos, tendo sido incluído na ocasião no grupo 1 da IARC (International Agency for Research on Cancer), classificação de 1998, mas continua no ano de 2012 com a mesma classificação (grupo 1) ) $^{37,38}$. Da mesma forma, o INSHT (Instituto Nacional de Seguridad e Higiene em el Trabajo), órgão do Ministerio de Trabajo y Assuntos Sociales-Espanha, no ano de 2002, considerou o OEt como substância carcinogênica de $2^{\mathrm{a}}$ categoria $(\mathrm{C} 2)$ por dispor de elementos suficientes evidenciados em estudos realizados em animais e outros estudos epidemiológicos realizados em profissionais expostos ao OEt em centrais de esterilização de hospitais e trabalhadores de indústrias químicas. Foi constatada associação entre a exposição a OEt e tumores de origem linfática e hematopoiética, fundamentalmente a leucemia linfoide e os linfomas não Hodgkin. Esses estudos também correlacionam aumento da ocorrência de abortos e a exposição profissional ao $\mathrm{OEt}^{37}$. Segundo o IARC-2008, após análise de estudos epidemiológicos (coorte e meta-análises) realizados nos EUA e Europa para verificação de possível associação entre exposição ao OEt e câncer de pâncreas, cérebro, estômago, mama e neoplasias do sistema hematopoiético, ainda não foi possível confirmar essa hipótese ${ }^{38}$. 
Embora não tenha sido estabelecida a relação entre a exposição ao OEt e neoplasias malignas, a maior incidência de malformações fetais em filhos de gestantes expostas a ele, assim como o maior risco de abortamentos espontâneos, são demonstrados em vários estudos ${ }^{39,40}$, demonstrando a capacidade mutagênica desse agente.

Para a Occupational Safety and Health Administration, um potencial carcinogênico ocupacional significa qualquer substância, combinação ou mistura de substâncias, que causam um aumento da incidência de neoplasias benignas ou malignas, ou uma substancial diminuição do período de latência entre a exposição e o aparecimento da doença em humanos ou em animais mamíferos de experimentação como resultado de exposição por via oral, respiratória ou dérmica, ou qualquer outra exposição que resulte na indução de tumor em um local diferente do local de administração. Esta definição também inclui qualquer substância que seja metabolizada em carcinogênicos ocupacionais pelos mamíferos ${ }^{41}$.

Para o estabelecimento de nexo entre a exposição ocupacional a um determinado agente e a ocorrência de doença é preciso em primeiro lugar determinar o risco. De acordo com o conceito, o risco possui três componentes: 1) potencial de perdas e danos; 2) a incerteza das perdas e danos; 3) a relevância das perdas e danos. Por isso sua expressão é: risco é igual à probabilidade de agravo à saúde, multiplicado pela magnitude das consequências em relação ao tempo ${ }^{42}$. No caso em tela, não foram fornecidas provas que esclarecessem o preciso contato do reclamante com o OEt, em que grau, em que concentração e em que tempo isso ocorreu. $\mathrm{O}$ fato de ele declarar que exercia as atividades laborais próximo ao local de carga e descarga do agente químico não é suficiente para estabelecer o grau de exposição.

Por definição, risco é a probabilidade de ocorrer o agravo à saúde, multiplicado pelo nível de exposição a que o trabalhador foi exposto ao agente, durante quanto tempo e qual a forma de exposição. A natureza cancerígena do agente também deve ser avaliada, assim como o grau de suscetibilidades dos órgãos e tecidos afetados. Também não foi possível identificar outros trabalhadores que adoeceram em decorrência do contato com o agente químico. A literatura médica não comprova, até o momento, a relação entre neoplasia de próstata e exposição ao OEt e, além disso, a neoplasia de próstata é de etiologia multifatorial e de alta incidência na população masculina, independentemente de exposição a algum agente químico. Portanto, o nexo ocupacional entre a exposição ao OEt e o câncer prostático não foi estabelecido.

\section{CONCLUSÃo}

Não foram encontrados estudos que confirmassem a associação entre a exposição ocupacional ao óxido de etileno e o desenvolvimento de câncer de próstata, portanto, o nexo ocupacional não foi considerado.

Peixinho RR; Miziara CSMG. Correlation between prostatic cancer and ethylene oxide exposure: occupational disease? Saúde, Ética \& Justiça. 2013;18(2):167-72.

\begin{abstract}
There are several articles in the medical literature that seek to verify if occupational exposure to chemical agents would occasion an increase in the incidence of prostate cancer, although prostate cancer is a multifactorial disease that likely involves both environmental and genetic factors. There is evidence that the prostate cancer risk varies most prominently with age, ethnicity, family history, and diet. The objective of this paper was to check if ethylene oxide could have a causal nexus to the development of prostatic cancer. The review articles published within the last twenty years have shown that there is still no consistent evidence that any chemical agent has an influence on the incidence of prostatic cancer. Consequently, to date there is no way of establishing if ethylene oxide has a causal nexus - to the development of this pathology.
\end{abstract}

KEYWORDS: Prostatic neoplasms/epidemiology; Risk factors; Ethylene oxide; Occupational exposure; Occupational cancer; Occupational risks.

\title{
REFERÊNCIAS
}

1. Brasil. Ministério da Saúde. Indicadores de mortalidade. [Internet]. 2004 [Acesso em 2013 março]. Disponível em : htpp//tabnet.datasus.gov.br/cgi/tabcgi.exe?idb2006/c10. def.

2. Brasil. Ministério da Saúde. Instituto Nacional do Câncer. Estimativas 2012 [Internet]. [Acesso em 2012 nov]. Disponível em: http://www.inca.gov.br/estimativa/2012/ estimativa20122111.pdf
3. Srougi M. Câncer da próstata. In: Srougi M, Simon SD, editores. Câncer urológico. $2^{\text {a }}$ ed. São Paulo: Platina; 1995. p. 281-359.

4. Kicinski M, Vangronsveld J, Nawrot TS. An epidemiological reappraisal of the familial aggregation of prostate cancer: a meta-analysis. PLoSOne 2011; 6(10): e27130.

5. Srougi M, Ribeiro LA, Piovesan AC, Colombo JR, Nesrallah A. Doenças da próstata. Rev Med (São Paulo) 
2008; 87(3): 166-77.

6. Hsing AW, Tsao L, Devesa SS. International trends and patterns of prostate cancer incidence and mortality. Int $\mathrm{J}$ Cancer. 2000; 85(1): 60-7.

7. Romero FR, Romero AW, Almeida RM, Oliveira FC Jr, Tambara Filho R. The significance of biological, environmental, and social risk factors for prostate câncer in a cohort study in Brazil. Int Braz J Urol. 2012; 38(6): 76978.

8. Goldqar DE, Easton DF, Cannon-Albright LA, Skolnick MH. Systematic population-based assessment of cancer risk in first-degree relatives of cancer probands. J Natl Cancer Inst. 1994; 86(21): 1600-8

9. Johns LE, Houlston RS. A systematic review and metaanalysis of familial prostate cancer risk. BJU Int. 2003; 91(9): 789-94.

10. Cao Y, Ma J. Body mass index, prostate cancer-specific mortality, and biochemical recurrence: a systematic review and meta-analysis. Cancer Prev Res (Phila). 2011; (4): 486501.

11. Hyde Z, Flicker L, McCaul KA, Almeida OP, Hankey GJ, Chubb SA, Yeap BB. Associations between testosterone levels and incident prostate, lung, and colorectal cancer. A population-based study. Cancer Epidemiol Biomarkers Prev. 2012; 21(8): 1319-29.

12. Ferreira V, Nardi AC. CP. In: Netto JR NR, organizador Urologia prática. São Paulo: Atheneu; 1999. p.237-46.

13. Reyes BL, Hanhan IWF. Câncer da próstata. In: Borden EC, Bosh FX, Campbell RJ, Coleman MP, Drinkwater NR, Eckhart S, organizadores. Manual de oncologia clínica. São Paulo: Fundação Oncocentro de São Paulo; 1997. p.311-6.

14. Barrios CH. CP. In: Murad AM, Katz A, organizadores. Oncologia, bases clínicas do tratamento. Rio de Janeiro: Guanabara Koogan; 1996. p.220-7.

15. Multiligner L, Ndong JR, OlivaA, BlanchetP. Environmental pollutants and prostate cancer: epidemiological data. Gynecol Obstet Fertil. 2008; 36 (9): 848-56.

16. Van Maele-Fabry G, Libotte V, Willens J, Lison D. Review and meta-analysis of risk estimates for prostate cancer in pesticide manufacturing workers. Cancer Causes Control. 2006; 17(4): 353-73.

17. Dennis LK. Meta-analysis for combining relative risks of alcohol consumption and prostate cancer. Prostate. 2000; 42(1): $56-66$

18. Cole P, Rodu B. Epidemilogic studies of chrome and cancer mortality: a series of meta-analyses. Regul Toxicol Pharmacol. 2005; 43(3): 225-31.

19. Stewart RE, Dennis LK, Dawson DV, Resnick MI. A metaanalysis of risk estimates for prostate cancer related to tire and rubber manufacturing operations. J Occup Environ Med. 1999; 41(12): 1079-84.

20. Schmitz-Drager BJ, Lummen G, Bismarck E, Fischer C. Prevention strategies for prostate cancer. Minerva Urol Nefrol. 2012; 64(4): 225-231.

21. Masko EM, Allot EH, Freedland SJ. The relationship between nutrition and prostate cancer: is more always better? Eur Urol. 2013; 63(5): 810-20.

22. United States of America. Department of Labor. OSHA Occupational Safety \& Health Administration. -Recognized chemicals as carcinogens or potential carcinogens for Hazard Communication purposes [Internet]. [Acesso em
2013 mai 08] Disponível em http://www.osha.gov/SLTC/ ethyleneoxide

23. Swaen,G, Burns C, Teta J, Bodner K, Keenan D, Bodnar C. Mortality study update of ethylene oxide workers in chemical manufacturing: A 15 year update. J Occup Environ Med. 2009; 51(6): 714-23

24. Teta MJ, Sielken RLJ, Valdez-Flores C. Ethylene oxide cancer risk assessment based on epidemiological data: application of revised regulatory guidelines. Risk Anal. 1999; 19(6): 1135-55.

25. Shore RE, Gardner MJ, Pannett B. Ethylene oxide: an assessment of the epidemiological evidence on carcinogenicity. Br J Ind Med. 1993; 50 (11): 971-9.

26. Mikoczy Z, Tinnerberg H, Bjork J, Albin M. Cancer incidence and mortality in Swedish sterilant workers exposed to ethylene oxide: update cohort study findings 1972-2006. Int J Environ Res Public Health. 2011; 8(6): 2009-19.

27. Julin B, Wolk A, Johansson JE, Anderson SO, Andrén O, Akesson A. Dietary cadmium exposure and prostate incidence: a population-based prospective cohort study. $\mathrm{Br}$ J Cancer. 2012; 107(5): 895-900.

28. Pan J, Plant JA, Voulvoulis N, Oates CJ, Ihlenfeld C. Cadmium levels in Europe: implications for human health. Environ Geochem Health. 2010; 32 (1): 1-12.

29. Sahmoun AE, Case LD, Jackson SA, Schwartz GG. Cadmium and prostate cancer: a critical epidemiologic analysis. Cancer Invest. 2005; 23(3): 256-63.

30. Multiligner L, Ndong JR, OlivaA, BlanchetP. Environmental pollutants and prostate cancer: epidemiological data. Gynecol Obstet Fertil 2008 Sep;36(9):848-56.

31. Van Maele-Fabry G, Libotte V, Willens J, Lison D. Review and meta-analysis of risk estimates for prostate cancer in pesticide manufacturing workers. Cancer Causes Control 2006 May; 17(4):353-73.

32. Jemal A, Siegel R, Xu J, Ward E. Cancer statistics 2010. CA Cancer J Clin. 2010; 60(5): 277-300.

33. Facina T. Estimativa 2012 - Incidência de Câncer no Brasil. Rev Bras Cancerol. 2011; 57(4): 557.

34. Heindenreich A, Bellmunt J, Bolla M, Joniau S, Mason M, Matveev V, et al. Guía de la EAU sobre el CP. Parte 1: cribado, diagnóstico y tratamento del câncer clínicamente localizado. Actas Urol Esp. 2011; 35(9): 501-14.

35. Smith DS, Carvalhal GS, Mager DE, Bullock AD, Catalona WJ. Use of lower prostate specific antigen cutoffs for prostate cancer in black and white man. J Urol. 1998; 160(5): 1734-8

36. Bratt $\mathrm{O}$. Hereditary prostate cancer: clinical aspects. J Urol 2002; 168(3): 906-13.

37. Peña AA, Garcia MIG. Óxido de etileno (Protocolos de vigilancia sanitaria específica) - Comisión de Salud Pública. Madrid: Ministério de Sanidad y Consumo. 2003.32p.

38. IARC - Monographs on the Evaluation of Carcinogenic Risks to Humans. Ethylene Oxide. 2008; 97: 206-87.

39. Rowland AS, Baird DD, Shore DL, Darden B, Wilcox AJ. Ethylene oxide exposure may increase the risk of spontaneous abortion, preterm birth, and postterm birth. Epidemiology. 1996; 7(4): 363-8.

40. Gresie-Brusin DF, Kielkowski D, Baker A, Channa K, Rees D. Occupational exposure to ethylene oxide during pregnancy and association with adverse reproductive 
Peixinho RR; Miziara CSMG. Correlação entre exposição ao óxido de etileno e câncer de próstata: doença ocupacional?

outcomes. Int Arch Occup Environ Health. 2007; 80(7): 559-65.

41. United States of America. Department of Labor. OSHA Occupational Safety \& Health Administration. Carcinogens - Safety and Health Topic [Internet]. [Acesso em 2013 jun]. Disponível em: https://www.osha.gov/SLTC/carcinogens

Recebido em: 03/06/2013

Aprovado em: 14/06/2013
42. Gondim GMM. Do Conceito de Risco ao da Precaução: entre determinismos e incertezas. Educação Profissional e Docência em Saúde: a formação e o trabalho do agente comunitário de saúde [Internet]. [Acesso em 2013 jun] Disponível em: http://www.epsjv.fiocruz.br/pdtsp/index. php?livro_id $=6 \&$ area_id $=2 \&$ capitulo_id $=77 \&$ autor $\mathrm{id}=\&$ arquivo $=$ ver_conteudo_2 